\title{
The Opinion of General Practitioners, Medical Students, and Other Medical Specialists on Palliative Care in Bulgaria
}

\author{
G. Foreva, ${ }^{1}$ R. Asenova, ${ }^{1}$ and M. Semerdjieva ${ }^{2}$ \\ ${ }^{1}$ Section General Medicine, Public Health Faculty, Medical University Plovdiv, 15A Vassil Aprilov Street, 4002 Plovdiv, Bulgaria \\ ${ }^{2}$ Department Health Care Management, Public Health Faculty, Medical University Plovdiv, 15A Vassil Aprilov Street, \\ 4002 Plovdiv, Bulgaria
}

Correspondence should be addressed to G. Foreva; gforeva@dir.bg

Received 3 June 2013; Accepted 10 December 2013; Published 30 January 2014

Academic Editors: G. Becker, D. Heigener, and C. Knapp

Copyright (c) 2014 G. Foreva et al. This is an open access article distributed under the Creative Commons Attribution License, which permits unrestricted use, distribution, and reproduction in any medium, provided the original work is properly cited.

\begin{abstract}
In Bulgaria, the patient is entitled to palliative care in case of incurable disease with an unfavourable prognosis. Palliative care is provided by the family doctor/GP and institutions. Literature on palliative care providing is scarce. The objective of the study was to investigate the opinion of general practitioners, medical students, and other medical specialists working in institutions on palliative care. Method. We have developed a structured questionnaire. Descriptive statistics have been calculated for all items. Differences between groups have been compared using $u$-criterion. Level of significance was $P<0.05$. Data has been analyzed using SPSS v. 16. Results. A total of 518 respondents completed the survey. Lack of appropriate organisation and financing has been pointed out by all participants. The GP's role in palliative care providing has been described as a contradictory one. The criteria on the basis of which the patients are eligible for palliative care have been arranged in the same way by all respondents, but GPs chose the longest temporal indicator. Quality assessment has not been applied. 2/3 of respondents demanded palliative care training. Conclusion. On the whole, the investigated groups differed to some extent in their opinion on palliative care both on conceptual and practical levels.
\end{abstract}

\section{Introduction}

In the last 20 years, demographic development in Bulgaria has been characterized by population decline, a low birth rate, a high mortality rate, and ageing population.

Health care reforms after 1989 have provided changes in the structure and management of the health system; GPs have been established as providers of primary care and gatekeepers; the main financial source has been obtained from compulsory health insurance contributions in National Health Insurance Fund (NHIF). In spite of that fact, citizens as well as medical professionals are dissatisfied and the need for further reform is pronounced, particularly in view of the low health status of the population.

The term palliative care includes both the philosophy and organization of an approach that increases the quality of health care in three directions: alleviation of suffering, communication improvement, and coordination of care in different settings. Palliative care in Bulgaria is underdeveloped regarding both community-based services and inpatient care; it has been provided by general practitioners, health institutions for out-of-hospital and hospital-based care, oncological health centres, and hospices and financed by compulsory health insurance. Moreover, the availability of palliative care facilities is insufficient. In most cases, patients tended to stay at family home, mainly because of financial reasons. Patients directly pay nursing homes. The only clinical path in palliative care is for oncological patients in terminal stages. Thus, in general, palliative care is not organized well, and the role of general practitioners and other medical and nonmedical specialists in providing it is not established. Researches in this field are insufficient and there is scarcity in the scientific literature. The objective of the study was to investigate the opinion of general practitioners, medical students, and other medical specialists working in institutions, on palliative care, focused on (1) organization, (2) GPs' role, (3) identification of patients, (4) quality, and (5) medical specialists' training needs in palliative care. 


\section{Methods}

We have developed a structured questionnaire, after the literature review, consisting of 30 items on investigated topics and questions related to sociodemographic data.

To validate the question a pilot survey of 30 medical specialists was conducted. One month later they were asked the same questions. The obtained high value of the coefficients of internal coordination Cronbach's alpha and coefficient of Spearman-Brown $(\mathrm{rsb}=0.952)$ and the low ones of item-total correlations $(R=0.426)$ confirmed the reliability and validity of the questionnaire.

Descriptive statistics (e.g., frequency, percentage) have been calculated for all items. Differences between groups have been compared using $u$-criterion. Level of significance has been accepted at $P<0.05$. Data has been analyzed using SPSS v.16.4.

2.1. Characteristics of Sample. The questionnaire was spread, once without remainder, among three groups of medical specialists which had been chosen in order to collect different points of view: (1) GPs, randomly selected $10 \%$ sample by the list on a national level (total contracts with NHIF in primary care in Bulgaria: 3963 for 2010); (2) all medical students in their last year at Medical University Plovdiv, the alumni of $2009 / 2010(N=97)$; and (3) all medical specialists working in the institutions in Plovdiv region who provide palliative care (employees in one hospital department and four hospices, $N=129)$.

\section{Results}

Responding rate was $82.75 \%$. The total number of analyzed questionnaires was 518. The sociodemographic characteristics of investigated groups have been presented in Table 1.

3.1. Organization of Palliative Care. Organizing and financing palliative care were pointed out as the greatest difficulties. Studied groups are defined as an adequate organizational form-specialized palliative care units (that should have the resources to provide care at home, as well as in hospital/hospice setting).

On the whole, participants shared the opinion that the home was the place preferred by the patients for palliative care. General practitioners considered home care to be of a greater significance, as compared to physicians from institutions $(P=0.011)$ and nurses $(P=0.016)$, but not to medical students $(P=0.15)$.

More than half of the general practitioners have chosen health insurance as an appropriate source of financing, whereas $23.55 \%$ of the group of other medical specialists have given a priority to the state budget 5 .

3.2. Place and Role of General Practitioners. Only $18.55 \%$ of the group of other medical specialists considered the GP as a coordinator. There was a significant difference in the standpoint of GPs (49.31\%) and the physicians working in institutions (14.32\%) regarding the coordinating role of
TABLE 1: Characteristics of sample.

\begin{tabular}{lc}
\hline & No. $(\%)$ \\
\hline Total & $\mathbf{5 1 8 ( 1 0 0 )}$ \\
GP & $337(65.05)$ \\
Medial students & $84(16.21)$ \\
Other medical specialists & $97(18.75)$ \\
$\quad$ Physicians & $43(8.30)$ \\
$\quad$ Nurses & $54(10.42)$ \\
Gender & \\
$\quad$ Male & $333(64.28)$ \\
Female & $185(35.72)$ \\
Age & \\
18-30 & $100(19.31)$ \\
31-40 & $102(19.69)$ \\
$41-50$ & $190(36.67)$ \\
51-60 & $104(20.08)$ \\
61-70 & $22(4.25)$ \\
GPs' practice location & \\
Urban 20 000 inhabitants & \\
Suburban less than 20 000 inhabitants & $206(61.12 \%)$ \\
Rural & $83(24.62 \%)$ \\
Patients' lists in general practices & $48(14.24 \%)$ \\
\hline
\end{tabular}

the GP, $P=0.001(u=6.16)$, and the nurse $(22.66 \%)$, $P=0.001(u=4.42)$.

Only $2.06 \%$ of the other medical specialists stated that they sustained active relations with the GP, whereas $88.65 \%$ expected the GP to get in touch with them.

The "ideal" team for palliative care was described. The highest percentage of the respondents pointed out the psychologist $(81.76 \%)$. The specialist in the disease profile $(81.21 \%)$, the nurse $(79.00 \%)$, the carer $(58.01 \%)$, the GP (56.35\%), and the social worker $(45.30 \%)$ were the basis of the team. The volunteers $(28.72 \%)$, the clergyman $(26.51 \%)$, and the dietitian $(22.65 \%)$ were stated at the end of this list. The psychologist was defined by the GPs and students as the person who had to break "the bad news" (24.30\%), whereas the other medical specialists pointed out in the first place the specialist in the disease profile (55.24\%), $P=0.032$. Part of the GPs $(56.37 \%)$ considered this to be their obligation, an opinion that was not accepted by their colleagues $(16.66 \%)$, $P=0.001$. Among the respondents who have chosen the psychologist as the most appropriate specialist to talk about death with the patient, the physicians from institutions stated this opinion to a greater degree significant from a statistical point of view, as compared to the GPs, $P=0.015$.

3.3. Identification of Patients in Need of Palliative Care. Comparing the criteria in identifying the patients for palliative care all participants arranged the criteria in one and the same way, namely, diagnosis, symptoms, and finally prognosis, $P=$ 0.094. According to a prognosis as the temporal criterion, 
other medical specialists pointed out the shortest period of three months, whereas the GPs have chosen the longest period-one year. A minimal part of the respondents have used written scales (e.g., Karnofsky performance scale) to assess the need of palliative care.

For assessment of pain and/or other principal symptoms, the comparison of the answers of other medical specialists showed that scales were more frequently used by those working in hospitals (35.05\%), as compared to those working in hospices $(6.18 \%), P<0.001(u=4.47)$. No significant difference was found in the utilization of such scales by GPs and other medical specialists, $P=0.079$.

3.4. Determination of the Quality of Palliative Care. As the most important aspect of the quality of life, GPs prioritized community support, whereas other medical specialists and students psychological aspects.

No significant difference was found regarding the frequency with which GPs and other medical specialists stated that they assessed the quality of life of their patients $(P=$ $0.530)$, which most frequently was done in a nonformal conversation.

3.5. Need of Training in Palliative Care/Medicine. Almost half of the respondents have taken a specialized course in palliative care. More trained in the area were the nurses as compared to GPs, $P<0.001(u=3.68)$. Almost $2 / 3$ of the respondents have been of the opinion that additional training in palliative care is necessary.

Identifying palliative care as a separate medical specialty was pointed out as necessary by $48.07 \%$ of the group of other medical specialists and students. No significant difference in the opinion of both groups studied was found regarding this matter, $P=0.083$. However, considering the subgroups, the GPs shared to a greater extent the opinion that palliative medicine should be taught as an individual specialty (40.46\%), as compared to the physicians working in institutions (23.80\%), $P<0.05(u=2.38)$.

\section{Discussion}

All respondents have agreed on the same difficulties in providing palliative care-the organization and financingand all of them have shared the opinion that specialized units for palliative care are needed.

The home is the place preferred by the patients for palliative care. As expected, GPs attached a greater importance to that preference, but the students attached an even greater importance to it. The tendency of deinstitutionalization is in agreement with the modern concept of palliative care.

In most countries, the word "hospice" is associated with a variety of financial sources, and it is not considered specifically to be a place for provision of palliative care only [1]. Filbet compared in their study different approaches to financing in relation to the place where palliative care was provided [2]. As far as the appropriate source of financing was concerned, GPs insisted on health insurance, whereas other medical specialists preferred the state budget.
Without directly denying the place occupied by the GPs, the other medical specialists and students considered their coordinating role as less important, which supplanted palliative care to hospitals. Coordinating function is basic for family doctors. In a study involving Belgian GPs, the latter reported that their cooperation with specialized palliative care teams was satisfactory [3]. In principle, palliative care is planned, ensuring a clear understanding of the role of each one included in the team [4]. GPs are familiar with the patient's context and specialized palliative care adds detailed advice to the treatment of symptoms and direct provision of care on the part of the nurses, including the spiritual aspects [5]. The relationship between those providing primary care and the palliative care specialists providing assistance in home setting as well has positive consequences because it improves the quality of medical care $[6,7]$. There is no continuity of care in our country, as well as established relations between the health care institutions, the social service units, and the psychological services.

In principle, the psychological aspect of palliative care is accepted as an obligatory part and is determined as the ability of medical specialists to encourage patients and family members to discuss their thoughts, feelings, and apprehensions. We live in a culture dominated by the cult of youth and to a certain extent the topic of death is associated with fear and is frequently pushed aside [8]. Almost half of the other medical specialists stated that they have not discussed with their patients matters related to death, although the process of dying is medicalized in modern societies.

Although other medical specialists arranged the criteria on the basis of which the patients are eligible for palliative care in the same way as GPs do, other medical specialists differed in their choice of a shorter period. At present there is a tendency to prolong the period of time. The studies show that in nononcological diseases in particular, apart from the diagnosis, a combination of criteria should be used as well, including performance status and clinical and laboratory data [9].

Assessment of the quality of life is an obligatory component of palliative care. Although the other medical specialists and students considered as the most important the psychological aspect of quality of life, its spiritual aspects remain in the last place for this group as well. The study of Alexandrova reported actual lack of clergymen in the teams providing palliative care. Provision of spiritual support was offered by certain institutions in cooperation with the local religious community [10]. Studies have been made in this area using scales among a large number of patients; for instance, the study of Kleeberg et al. involved almost 5,000 patients [11]. Our study confirms the fact that quality of life is not systematically studied in either general practices or institutions.

Communication with the patients receiving palliative care is associated not only with the existential questions of disease and death, but also with the whole process of suffering/being ill. The health care system is focused on aggressive treatment approaches, and suffering does not receive sufficient attention [12]. Communicative skills are acquired not only by accumulating professional experience, 
but also through guided training. These skills have bearing not only on the communication between physician and patient, physician and family members, but also on the interdisciplinary relations within the team and with the community [13].

All studied groups reported a considerable need of training in palliative care. This is confirmed by a number of studies. Investigating the opinion of specializing physicians in surgery and general practice, Celso et al. found that both groups pointed out the importance of training in palliative care [14]. Medical schools in the USA include an obligatory basis on the so-called "care at the end of life" in their syllabus [15]. The studies on such type of training in Europe show that it is fragmented, not assessed formally, in spite of the EU recommendations given in 1993, that training in palliative care should be an integral part of training in medicine at a student level, and that students should sit an examination [16].

4.1. Limitation of the Study. On the base of the literature review, we have developed a questionnaire, trying to explore challenging aspects in palliative care field. The study has been done with researchers' questionnaire, because of the lack of appropriate and validated instruments in Bulgaria for research.

The sample of the study has been recruited both on national (representative sample of GPs) and regional levels. Having in mind that different medical specialists have an impact on palliative care, we have widened the investigated group, being able to ensure a comprehensive sample regarding students and medical specialists working in hospices and hospital palliative department on regional levels.

\section{Conclusion}

Lack of an appropriate organization and financing was pointed out by all participants in the study. The attitude among the medical community regarding the GPs' role and place in Bulgarian health care system is contradictory, including their role in palliative care providing. The criteria on the basis of which the patients are eligible for palliative care were arranged, diagnosis, symptoms, and prognosis, in the same way by all respondents, but GPs have chosen the longest temporal indicator of a year. Quality assessment is more frequently applied in the health care practice. More needs to be done, taking into account the answers to the questionnaire in this field. Additional palliative care training has been demanded.

On the whole, the GPs, medical students, and other medical specialists, working in institutions where palliative care is given, differed to some extent in their opinion on palliative care both on conceptual and practical levels.

\section{Conflict of Interests}

The authors declared no potential conflict of interests with respect to the research, authorship, and/or publication of this article.

\section{References}

[1] N. Milićević, "The hospice movement: history and current worldwide situation," Archive of Oncology, vol. 10, no. 1, pp. 2931, 2002.

[2] M. Filbet, "Putting the plans for French palliative care into action," European Journal of Palliative Care, vol. 10, no. 1, pp. 24-26, 2003.

[3] M. Desmedt and H. Michel, "Palliative home care: improving co-operation between the specialist team and the family doctor," Supportive Care in Cancer, vol. 10, no. 4, pp. 343-348, 2002.

[4] D. C. Currow and E. M. Nightingale, "A planning guide': developing a consensus document for palliative care service provision," Medical Journal of Australia, vol. 179, no. 6, supplement, pp. S23-S25, 2003.

[5] J. P. Griffin, K. A. Koch, J. E. Nelson, and M. E. Cooley, "Palliative care consultation, quality-of-life measurements, and bereavement for end-of-life care in patients with lung cancer: ACCP evidence-based clinical practice guidelines (2nd Edition)," Chest, vol. 132, no. 3, supplement, pp. 404S-422S, 2007.

[6] D. Goldschmidt, L. Schmidt, A. Krasnik, U. Christensen, and M. Groenvold, "Expectations to and evaluation of a palliative home-care team as seen by patients and carers," Supportive Care in Cancer, vol. 14, no. 12, pp. 1232-1240, 2006.

[7] S. A. Murray and J. Y. Kok, "Internationally, it is time to bridge the gap between primary and secondary healthcare services for the dying," Annals of the Academy of Medicine Singapore, vol. 37, no. 2, pp. 142-144, 2008.

[8] R. McKechnie, R. MacLeod, and S. Keeling, "Facing uncertainty: the lived experience of palliative care," Palliative and Supportive Care, vol. 5, no. 3, pp. 255-264, 2007.

[9] E. Fox, K. Landrum-McNiff, Z. Zhong, N. V. Dawson, A. W. Wu, and J. Lynn, "Evaluation of prognostic criteria for determining hospice eligibility in patients with advanced lung,heart, or liver disease," The Journal of the American Medical Association, vol. 282, no. 17, pp. 1638-1645, 1999.

[10] S. Alexandrova, Hospice and Palliative Care, Global Practice and Bulgarian Experience Medical University Publishing House, Pleven, Bulgaria, 2009, (Bulgarian).

[11] U. R. Kleeberg, P. Feyer, W. Günther, and M. Behrens, "Patient satisfaction in outpatient cancer care: a prospective survey using the PASQOC questionnaire," Supportive Care in Cancer, vol. 16, no. 8, pp. 947-954, 2008.

[12] S. Daneault, V. Lussier, S. Mongeau et al., "Primum non nocere: could the health care system contribute to suffering? In-depth study from the perspective of terminally ill cancer patients," Canadian Family Physician, vol. 52, no. 12, pp. 1574-1575, 2006.

[13] J. Kleja, I. Filipczak-Bryniarska, and J. Wordliczek, "Communication in palliative care," Advances in Palliative Medicine, vol. 8, no. 4, pp. 147-153, 2009.

[14] B. G. Celso, D. Graham, J. J. Tepas, S. Meenrajan, and M. A. Schinco, "Competence in palliative and end of life care-general surgery and family medicine residencies," Advances in Palliative Medicine, vol. 9, no. 1, pp. 3-9, 2010.

[15] D. A. Seamark, C. J. Seamark, and D. M. G. Halpin, "Palliative care in chronic obstructive pulmonary disease: a review for clinicians," Journal of the Royal Society of Medicine, vol. 100, no. 5, pp. 225-233, 2007.

[16] M. Lloyd-Williams and D. M. MacLeod, "A systematic review of teaching and learning in palliative care within the medical undergraduate curriculum," Medical Teacher, vol. 26, no. 8, pp. 683-690, 2004. 


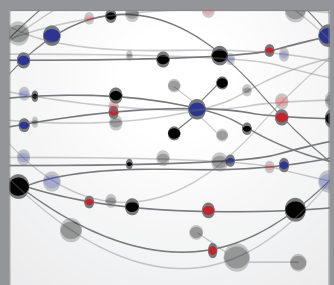

The Scientific World Journal
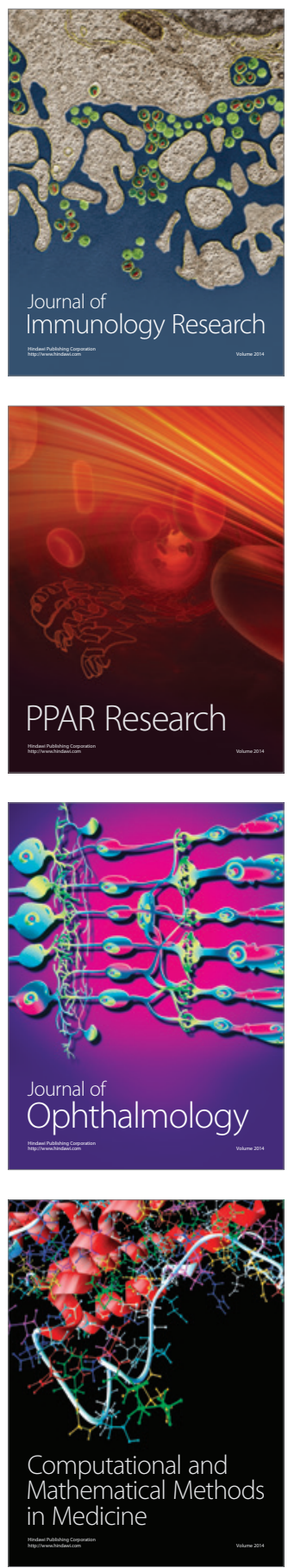

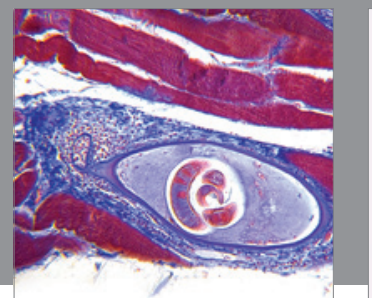

Gastroenterology

Research and Practice
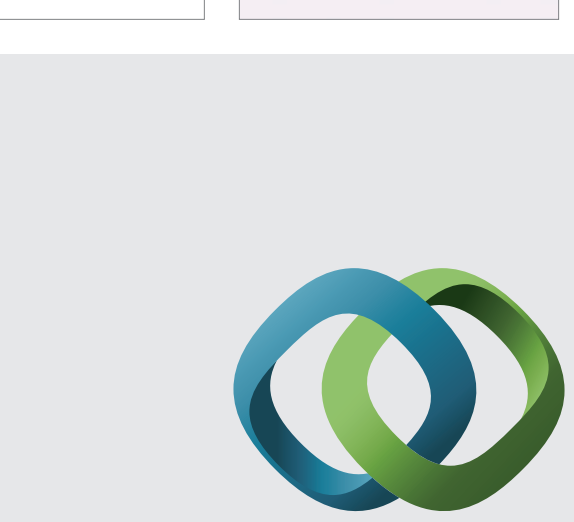

\section{Hindawi}

Submit your manuscripts at

http://www.hindawi.com
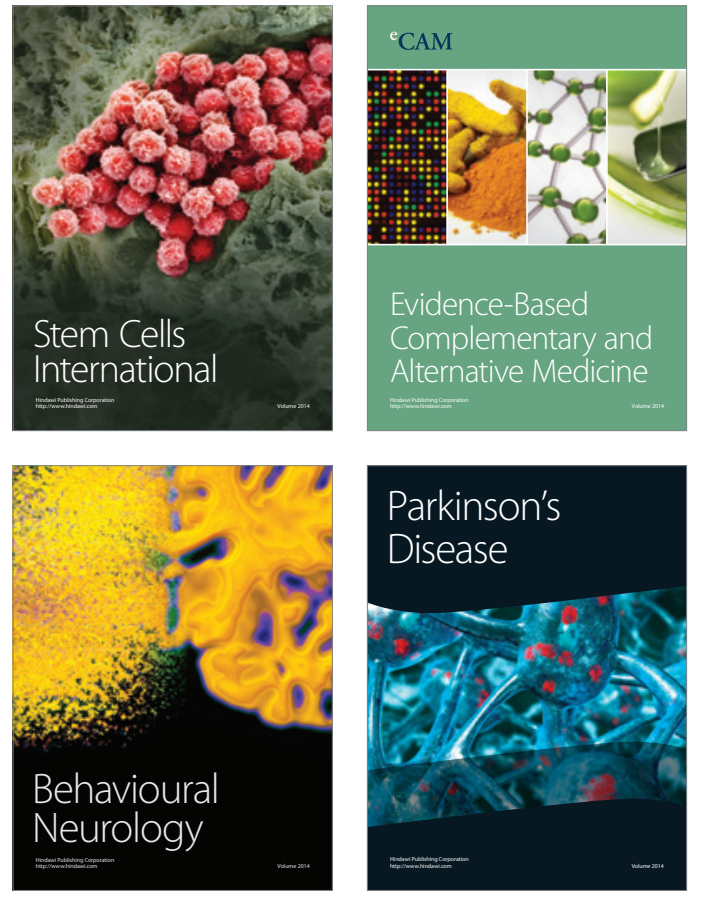
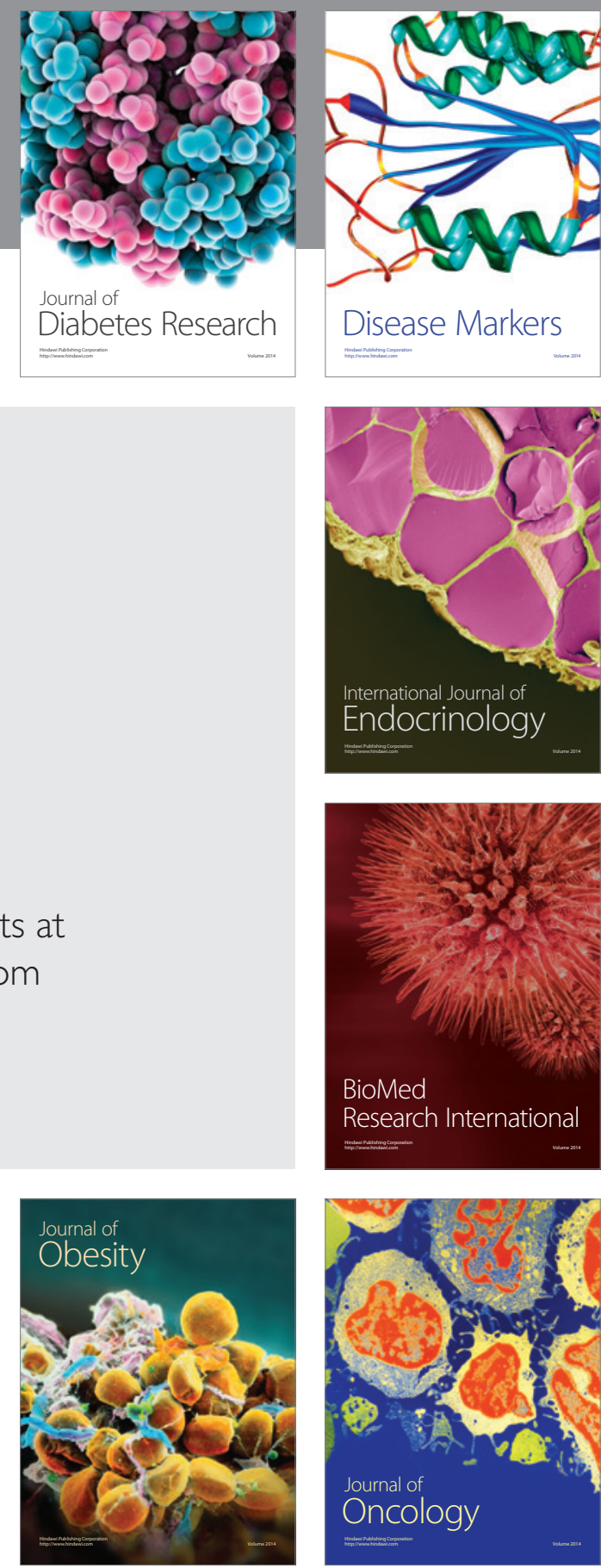

Disease Markers
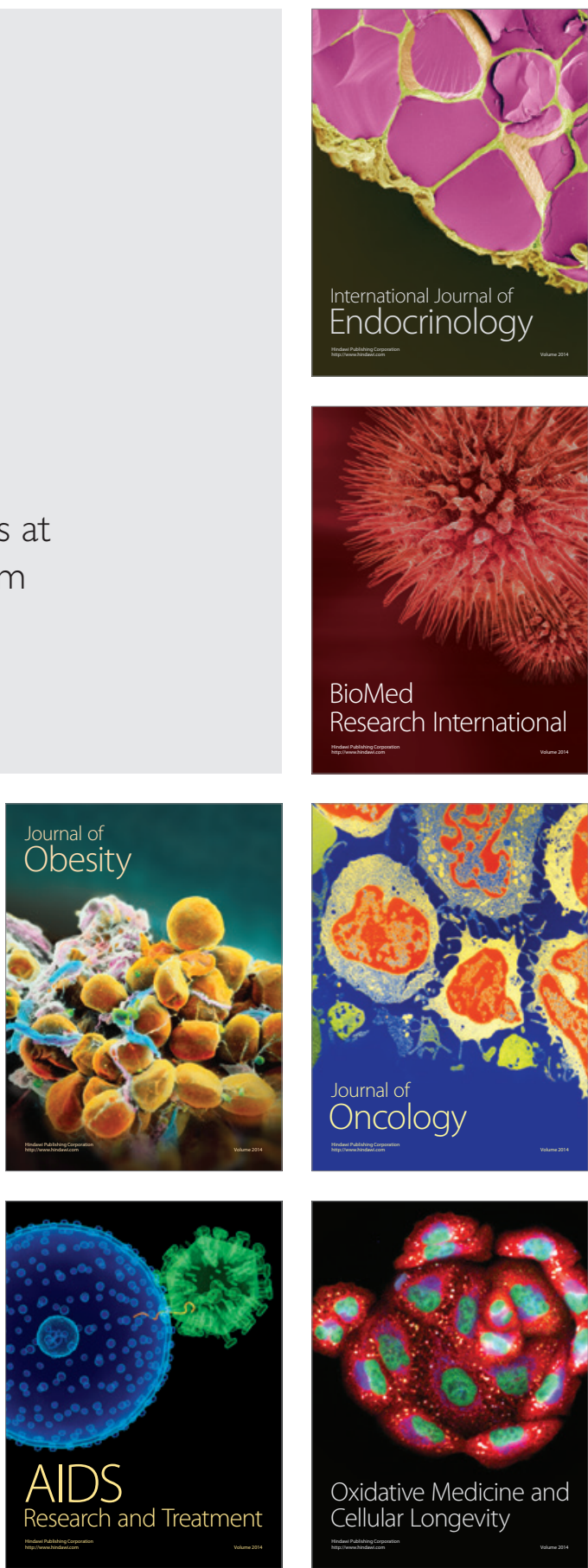